\title{
Universal Metaplectic Structures and Geometric Quantization
}

\author{
Michael Forger and Harald Hess* \\ Institut für Theoretische Physik, Freie Universität Berlin, D-1000 Berlin 33
}

\begin{abstract}
The recently developed concepts of generalized and universal spin structures are carried over from the orthogonal to the symplectic and unitary cases. It turns out that the analogues of $S p i{ }^{c}$-structures, namely the $M p^{c}$ structures and $M U^{c}$-structures, are sufficient to avoid topological obstructions to their existence. It is indicated how this fact can be used in the geometric quantization of certain suitably polarized symplectic manifolds with arbitrary second Stiefel-Whitney class, where the usual Kostant-Souriau quantization scheme breaks down.
\end{abstract}

\section{Introduction}

In field theory, an important criterion for a Riemannian manifold $M^{1}$ to be a reasonable model of space-time is that it admit spinors [7]. The conventional method of dealing with this problem is to require that $M$ has a spin structure [15]. The bundle of spinors over $M$ is then the complex vector bundle associated to the corresponding principal bundle of spin frames over $M$ and the spin representation of its structure group Spin on the space of spinors $S$.

On the other hand, in geometric quantization, one can apply the same idea to a symplectic manifold $M$ and require that $M$ has a metaplectic structure. The bundle of symplectic spinors over $M$ is then the complex vector bundle associated to the corresponding principal bundle of metaplectic frames over $M$ and the metaplectic representation of its structure group $M p$ on the space of symplectic spinors $S$. In some sense, this contains the complex line bundle of pure symplectic spinors over $M$, which provides for an explicit realization of the bundle of half-forms and the $B K S$ pairing and hence plays an important role in geometric quantization. For more details, we refer to [14].

1 For simplicity, we work with Riemannian $(++\ldots+)$ rather than Lorentzian $(+-\ldots-)$ manifolds $M$ and assume them to be even-dimensional, but this does not affect our arguments concerning spin and spinors. We also tacitly assume $M$ to be oriented (so that in particular, the first Stiefel-Whitney class $w_{1}(M) \in H^{1}\left(M, \mathbb{Z}_{2}\right)$ of $M$ vanishes $)$

* Supported by the Studienstiftung des deutschen Volkes 
Finally, consider a (n almost) hermitian manifold $M$ and require that $M$ has a metaunitary structure. Then one has the complex line bundle associated to the corresponding principal bundle of metaunitary frames over $M$ and the representation of its structure group $M U$ on $\mathbb{C}$ defined as the square root of the determinant. This line bundle is a square root of (the dual of) the canonical line bundle of $M$ and is used in various contexts; see e.g. [1,2].

There is a topological obstruction to the existence of spin structures on a Riemannian manifold $M$, of metaplectic structures on a symplectic manifold $M$, and of metaunitary structures on a (n almost) hermitian manifold $M$ : In all three cases, such structures will exist iff the second Stiefel-Whitney class $w_{2}(M) \in H^{2}\left(M, \mathbb{Z}_{2}\right)$ of $M$ vanishes. Moreover, whenever $w_{2}(M)=0$, the set of inequivalent spin structures resp. metaplectic structures resp. metaunitary structures on $M$ can be brought into a $1-1$ correspondence with the first cohomology group $H^{1}\left(M, \mathbb{Z}_{2}\right)$ of $M$ with $\mathbb{Z}_{2}$-coefficients. See $[5,15,14,1,10]$.

However, there exist interesting manifolds, such as the complex projective spaces $P_{n}(\mathbb{C})$ with $n$ even, whose second Stiefel-Whitney class is nonzero and for which the above methods do not apply. In particular, in geometric quantization, where $P_{n}(\mathbb{C})$ appears as the quotient of energy surfaces for the $(n+1)$-dimensional harmonic oscillator, the conventional Kostant-Souriau quantization procedure breaks down if $n$ is even.

\section{Generalized Spin, Metaplectic and Metaunitary Structures}

To resolve this dilemma, let us introduce the concept of a generalized spin structure $[8,18]$ and the analogous concepts of a generalized metaplectic structure and generalized metaunitary structure: Fix a Lie group $G$ which contains $\mathbb{Z}_{2}$ as a discrete central subgroup, and consider the extension $\bmod \mathbb{Z}_{2}$

$$
\operatorname{Spin}^{G}(2 n)=\operatorname{Spin}(2 n) \times_{\mathbb{Z}_{2}} G
$$

of the spin group $\operatorname{Spin}(2 n)$ by $G$, where $2 n=\operatorname{dim} M$. We have the commutative diagram

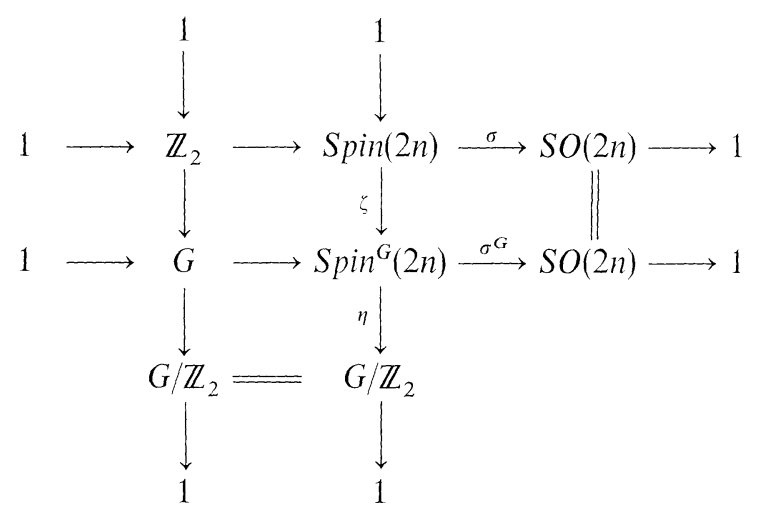


with exact rows and columns, where $\sigma$ is the double covering of $\operatorname{SO}(2 n)$ by $\operatorname{Spin}(2 n)$, and the homomorphisms $\sigma^{G}, \zeta, \eta$ are given by

$$
\sigma^{G}[A, g]=\sigma(A), \quad \zeta(A)=[A, 1], \quad \eta[A, g]=[g]
$$

for $A \in \operatorname{Spin}(2 n), g \in G$, where the square brackets indicate taking equivalence classes $\bmod \mathbb{Z}_{2}$. Then a generalized spin structure on $M$ is a $\operatorname{Spin}^{G}(2 n)$-structure on $M$. Applying the homomorphism $\eta$ to the (transition functions of the) principal bundle of generalized spin frames over $M$, we obtain a principal $\left(G / \mathbb{Z}_{2}\right)$-bundle over $M$, which we shall call the canonical prolongation of the given generalized spin structure (to $G / \mathbb{Z}_{2}$ ). For the symplectic case, we replace $S O(2 n)$ by $S p(2 n, \mathbb{R})$, $\operatorname{Spin}(2 n)$ by the double covering group $M p(2 n, \mathbb{R})$ of $\operatorname{Sp}(2 n, \mathbb{R})$, and $\operatorname{Spin}^{G}(2 n)$ by

$$
M p^{G}(2 n, \mathbb{R})=M p(2 n, \mathbb{R}) \times_{\mathbb{Z}_{2}} G,
$$

while for the unitary case, we replace $S O(2 n)$ by $U(n)$, $\operatorname{Spin}(2 n)$ by the double covering group $M U(n)$ of $U(n)$, and $\operatorname{Spin}^{G}(2 n)$ by

$$
M U^{G}(n)=M U(n) \times_{\mathbb{Z}_{2}} G,
$$

using the same notation for the homomorphisms. Moreover, fix a representation $T: G \rightarrow A u t(V)$ of $G$ on a complex vector space $V$ taking the nontrivial element of $\mathbb{Z}_{2} \subset G$ to $-\mathrm{id}_{V}$. Then the direct product of $T$ with the spin representation $\operatorname{Spin}(2 n) \rightarrow \operatorname{Aut}(S)$ yields a representation $\tilde{T}: \operatorname{Spin}^{G}(2 n) \rightarrow A u t(\tilde{S})$ of $\operatorname{Spin}^{G}(2 n)$ on the space $\tilde{S}=S \otimes V$ of "spinors with coefficients in $V$ ". Hence given a generalized spin structure on $M$, we can define the bundle of generalized spinors over $M$ as the complex vector bundle associated to the corresponding principal bundle of generalized spin frames over $M$ and the representation $\tilde{T}$ of its structure group $\operatorname{Spin}^{G}(2 n)$. For the symplectic case, we replace $\operatorname{Spin}(2 n)$ by $M p(2 n, \mathbb{R})$ and $\operatorname{Spin}^{G}(2 n)$ by $M p^{G}(2 n, \mathbb{R})$ to obtain the space $\tilde{S}=S \otimes V$ of "symplectic spinors with coefficients in $V$ " and the bundle of generalized symplectic spinors.

As a well-known example $[3,2,11]$ to be discussed extensively below, we consider the $S p i^{c}$-structures, $M p^{c}$-structures and $M U^{c}$-structures, where $G=U(1)$, $G / \mathbb{Z}_{2}=U(1)$, the homomorphism $\eta$ is the squaring map in the second component, and $T$ is the defining representation of $G=U(1)$ on $V=\mathbb{C}$. The particular feature here is that $\tilde{S} \cong S$ since $S$ is a complex vector space anyway, so that the generalized (symplectic) spinors are just ordinary ones.

In general, there is still a topological obstruction to the existence of generalized spin structures on a Riemannian manifold $M$, of generalized metaplectic structures on a symplectic manifold $M$, and of generalized metaunitary structures on a(n almost) hermitian manifold $M$, but it involves a weaker condition than the vanishing of the second Stiefel-Whitney class. For example, if $G=U(1)$, then in all three cases, such structures will exist iff the second Stiefel-Whitney class $w_{2}(M) \in H^{2}\left(M, \mathbb{Z}_{2}\right)$ of $M$ is the reduction $\bmod 2$ of an integral cohomology class, i.e. iff there exists a cohomology class $c_{1} \in H^{2}(M, \mathbb{Z})$ such that $w_{2}(M)$ is the image of $c_{1}$ under the homomorphism $H^{2}(M, \mathbb{Z}) \rightarrow H^{2}\left(M, \mathbb{Z}_{2}\right)$ of cohomology groups induced by the homomorphism $\mathbb{Z} \rightarrow \mathbb{Z}_{2}$ of coefficient groups $[2,11]$. This raises the following natural question: Can we choose $G$ in such a way as to make this obstruction vanish altogether? We shall show that this can indeed be done, and that moreover there 
exists a distinguished $\operatorname{Spin}^{G}$-structure resp. $M p^{G}$-structure resp. $M U^{G}$-structure on any $M$, which we shall call the universal one.

From the aforementioned criterion, it is clear that in the symplectic and unitary cases, the topological obstruction will already vanish for $G=U(1)$ : In fact, the symplectic case reduces to the unitary case since $U(n)$ is the maximal compact subgroup of $S p(2 n, \mathbb{R})$, so that any symplectic manifold becomes an almost hermitian manifold with respect to some compatible almost complex structure. For the statement in the unitary case (where the tangent bundle of $M$ is a complex vector bundle), choose $c_{1}$ to be the first Chern class of $M$ (which is what the notation above was supposed to suggest). However, this argument is not sufficient for our purposes since we are also interested in singling out specific $M p^{c}$-structures and $M U^{c}$-structures, respectively, and in studying connections.

The idea of the construction is very simple [4]: All we need is a homomorphism $\chi: \operatorname{Spin}(2 n) \rightarrow G$ taking the nontrivial element of $\mathbb{Z}_{2}$ in $\operatorname{Spin}(2 n)$ to the nontrivial element of $\mathbb{Z}_{2}$ in $G$. Indeed, given $\chi$, we define a homomorphism

$$
\begin{aligned}
\chi^{\prime}: \operatorname{Spin}(2 n) & \longrightarrow \operatorname{Spin}^{G}(2 n) \\
A & \longrightarrow[A, \chi(A)]
\end{aligned}
$$

and observe that $\chi^{\prime}$ factors through the covering homomorphism $\sigma: \operatorname{Spin}(2 n) \rightarrow \operatorname{SO}(2 n)$ to yield a homomorphism $\chi^{\prime \prime}: \operatorname{SO}(2 n) \rightarrow \operatorname{Spin}^{G}(2 n)$ which is a right inverse to the homomorphism $\sigma^{G}: \operatorname{Spin}^{G}(2 n) \rightarrow S O(2 n)$. Therefore, applying $\chi^{\prime \prime}$ to the (transition functions of the) principal $S O(2 n)$-bundle of orthonormal frames of a Riemannian manifold $M$, we obtain the desired principal $\operatorname{Spin}^{G}(2 n)$ bundle of universal spin frames of $M$. Moreover, under the induced $\chi^{\prime \prime}$-equivariant homomorphism $\tilde{\chi}^{\prime \prime}$ of principal bundles over $M$, a given metric connection in $M$ i.e. a principal connection in the bundle of orthonormal frames of $M-\operatorname{maps} t^{2}$ a well-defined principal connection in the bundle of universal spin frames of $M$. In particular, the Levi-Cività connection in $M$-i.e. the unique metric connection in $M$ with vanishing torsion [12] - maps to what we shall call the Levi-Cività connection in the bundle of universal spin frames of $M$. Obviously, this technique also works in the symplectic and unitary cases, and we use the same notation for the homomorphisms.

To get an impression of how large $G$ has to be chosen in order for the desired homomorphism $\chi$ to exist, note that $G$ has to contain the image of $\chi$ as a Lie subgroup $H$ whose Lie algebra $\mathfrak{h}$ is nontrivial. (Otherwise, $H$ would be discrete and hence trivial since $\operatorname{Spin}(2 n), M p(2 n, \mathbb{R})$ and $M U(n)$ are connected, and $\chi$ could not take the nontrivial element of $\mathbb{Z}_{2}$ there to the nontrivial element of $\mathbb{Z}_{2}$ in $G$.) This leaves the following possibilities:

a) $\operatorname{Spin}(2) \cong U(1): \mathfrak{h}=u(1) \cong \mathbb{R}$.

b) $\operatorname{Spin}(4) \cong S U(2) \times S U(2): \mathfrak{h}=s u(2)$ or $\mathfrak{h}=s u(2) \oplus s u(2)$. This important special case is analyzed in [4] and was one of the starting points of the present work.

c) $\operatorname{Spin}(2 n)(n \neq 1, n \neq 2)$ resp. $M p(2 n, \mathbb{R})$ : These Lie groups are simple, so that $\mathfrak{h}=\operatorname{spin}(2 n)=\operatorname{so}(2 n)$ resp. $\mathfrak{h}=m p(2 n, \mathbb{R})=\operatorname{sp}(2 n, \mathbb{R})$.

d) $M U(n): \mathfrak{h}=u(1) \cong \mathbb{R}$ or $\mathfrak{h}=s u(n)$ or $\mathfrak{h}=u(n)$.

2 We use the concept of mappings of connections as explained in [12, p. 79f.] 
In particular, we see explicitly that in the unitary case, it is sufficient to work with $G=U(1)$ because we have the homomorphism

$$
\chi: M U(n) \rightarrow U(1)
$$

at our disposal which is the square root of the determinant. In other words, we have the commutative diagram

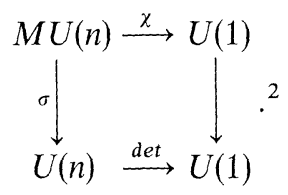

As before, we define a homomorphism

$$
\begin{aligned}
\chi^{\prime}: M U(n) & \longrightarrow M U^{c}(n) \\
A & \longmapsto[A, \chi(A)]
\end{aligned}
$$

and observe that $\chi^{\prime}$ factors through the covering homomorphism $\sigma: M U(n) \rightarrow U(n)$ to yield a homomorphism $\chi^{\prime \prime}: U(n) \rightarrow M U^{c}(n)$ which is a right inverse to the homomorphism $\sigma^{c}: M U^{c}(n) \rightarrow U(n)$. Therefore, applying $\chi^{\prime \prime}$ to the (transition functions of the) principal $U(n)$-bundle of unitary frames of a (n almost) hermitian manifold $M$, we obtain the desired principal $M U^{c}(n)$-bundle of universal metaunitary frames of $M$. Moreover, under the induced $\chi^{\prime \prime}$-equivariant homomorphism $\tilde{\chi}^{\prime \prime}$ of principal bundles over $M$, a given almost complex metric connection in $M$-i.e. a principal connection in the bundle of unitary frames of $M-$ maps $t^{2}$ a well-defined principal connection in the bundle of universal metaunitary frames of $M$. In particular, the hermitian connection in $M$ - i.e. the unique almost complex metric connection in $M$ with connection form of type $(1,0)$ [12] - maps to what we shall call the hermitian connection in the bundle of universal metaunitary frames of $M$. Finally, we have the commutative diagram

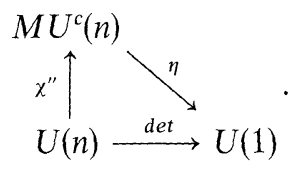

In the orthogonal and symplectic cases, on the other hand, the choice $G=U(1)$ has to be combined with using reductions of structure group: In fact, notice first that the embedding $\kappa: U(n) \rightarrow S O(2 n)$ resp. $\kappa: U(n) \rightarrow S p(2 n, \mathbb{R})$ lifts to an embedding $\hat{\kappa}: M U(n) \rightarrow \operatorname{Spin}(2 n)$ resp. $\hat{\kappa}: M U(n) \rightarrow M p(2 n, \mathbb{R})$ such that the diagram

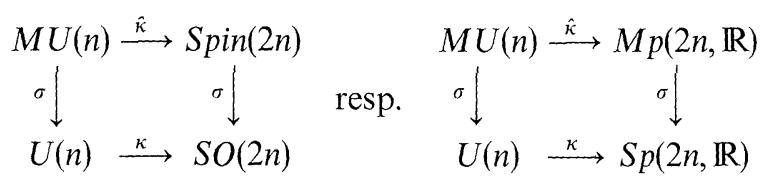

is commutative. (In fact, as double covering groups, $\operatorname{Spin}(2 n), M p(2 n, \mathbb{R})$ and $M U(n)$ corresponds to certain subgroups - namely $\{1\}, 2 \mathbb{Z}$ and $2 \mathbb{Z}$ - of the fundamental groups $\pi_{1}(S O(2 n))=\mathbb{Z}_{2}, \pi_{1}(S p(2 n, \mathbb{R}))=\mathbb{Z}$ and $\pi_{1}(U(n))=\mathbb{Z}$, respectively. But $\kappa$ induces a homomorphism $\kappa_{*}$ of fundamental groups taking $2 \mathbb{Z}$ to $\{1\}$ 
resp. to $2 \mathbb{Z}$, so that by a general theorem $\kappa$ lifts to a homomorphism $\hat{\kappa}$ which is then easily seen to have trivial kernel.) Hence $\kappa$ also induces an embedding

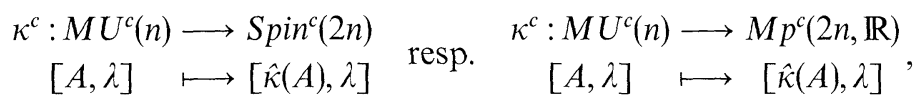

and we obtain the commutative diagram

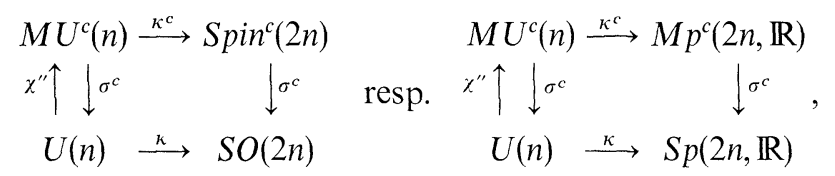

where the composition $\kappa^{c} \diamond \chi^{\prime \prime}$ is precisely the homomorphism introduced in [3]. Finally, we have the commutative diagram
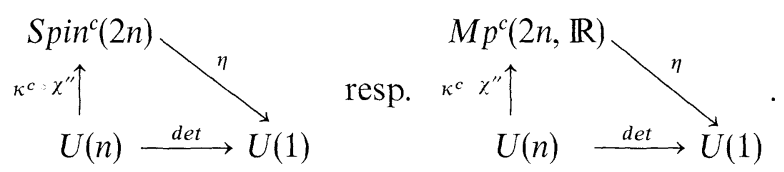

Now we assume we are given a Riemannian resp. symplectic manifold $M$, together with a compatible almost complex structure turning it into an almost hermitian manifold, so that the bundle of orthonormal resp. symplectic frames of $M$ has the bundle of unitary frames of $M$ as a distinguished reduction of structure group from $S O(2 n)$ resp. $S p(2 n, \mathbb{R})$ to $U(n)$. Applying $\kappa^{c} \circ \chi^{\prime \prime}$ to the (transition functions of the) latter, we obtain a canonical $S p i n^{c}$-structure resp. $M p^{c}$-structure on $M$, which we shall call the hermitian one, and which has the universal $M U^{c}$-structure on $M$ as a distinguished reduction of structure group from $\operatorname{Spin}^{c}(2 n)$ resp. $M p^{c}(2 n, \mathbb{R})$ to $M U^{c}(n)$. The canonical prolongation (to $U(1)$ ) of all these - as a principal $U(1)$ bundle, or equivalently, a hermitian complex line bundle over $M$ - is, due to (2.10) and (2.14), just the determinant bundle $\Lambda^{n} T M$ of $M$, i.e. the dual of the canonical line bundle of $M$, with first Chern class

$$
c_{1}=c_{1}\left(\Lambda^{n} T M\right)=c_{1}(T M)=c_{1}(M) .
$$

Moreover, under the induced $\left(\kappa^{c} \circ \chi^{\prime \prime}\right)$-equivariant resp. det-equivariant homomorphisms $\widetilde{\kappa^{c} \chi^{\prime \prime}}$ resp. det of principal bundles over $M$, the hermitian connection in $M$ - as a principal connection in the bundle of unitary frames of $M$ - maps to ${ }^{2}$ what we shall call the hermitian connection in the bundle of hermitian Spinc-frames resp. $M p^{c}$-frames resp. in the determinant bundle of $M$. In particular, the latter is the unique metric connection in the hermitian complex line bundle $\Lambda^{n} T M$ with curvature $\frac{i}{2} \varrho$, where $\varrho$ is the Ricci form of $M[12]$, and $c_{1}=c_{1}(M)$ is represented by the 2 -form $-\frac{1}{4 \pi} \varrho$.

We still have to discuss to what extent the constructions of the previous paragraph depend on the choice of the compatible almost complex structure. Note that if $M$ is a Riemannian resp. symplectic manifold and $P$ is its orthonormal resp. symplectic frame bundle, a compatible almost complex structure $J$ on $M$ can be 
identified with a reduction of structure group $Q$ of $P$ from $S O(2 n)$ resp. $S p(2 n, \mathbb{R})$ to $U(n) ; Q$ being a principal $U(n)$-bundle contained as a subbundle in the principal $S O(2 n)$-bundle resp. $S p(2 n, \mathbb{R})$-bundle $P$ (over $M$ ). Now in the orthogonal case, such a reduction of $P$ need neither exist nor be unique (up to an isomorphism of principal $U(n)$-bundles over $M$ ): As counterexamples, note that $M=S^{2 n}$ does not admit any compatible almost complex structure if $n \neq 1,3$ [12], while checking first Chern classes shows that $M=P_{n}(\mathbb{C})$ admits two inequivalent almost complex structures, $J$ and $-J$, (inducing the same orientation) if $n$ is even. In the symplectic case, on the other hand, such a reduction of $P$ always exists and is unique (up to an isomorphism of principal $U(n)$-bundles over $M$ ) because $U(n)$ is a maximal compact subgroup of $S p(2 n, \mathbb{R})$. Therefore, on a symplectic manifold $M$, a hermitian $M p^{c}$-structure always exists and is unique up to an isomorphism of principal $M p^{c}(2 n, \mathbb{R})$-bundles over $M$; unfortunately, this does not mean that it is unique as an $M p^{c}$-structure on $M$.

In fact, let $J_{1}$ and $J_{2}$ be two compatible almost complex structures on $M$ defining two reductions $Q_{1}$ and $Q_{2}$ of $P$ as above, and let $\tilde{P}_{k}=Q_{k} \times_{U(n)} M p^{c}(2 n, \mathbb{R})$ be the associated principal $M p^{c}(2 n, \mathbb{R})$-bundles and

$$
\begin{gathered}
\tilde{\sigma}_{k}^{c}: \tilde{P}_{k} \longrightarrow P \\
{[q,[A, \lambda]] \mapsto q \cdot \sigma(A)}
\end{gathered} \quad\left(q \in Q_{k}, A \in M p(2 n, \mathbb{R}), \lambda \in U(1)\right)
$$

the principal bundle homomorphisms defining the two corresponding hermitian $M p^{c}$-structures on $M(k=1,2)$. If $f: Q_{1} \rightarrow Q_{2}$ is an isomorphism of principal $U(n)$ bundles over $M$, it induces an isomorphism $\tilde{f}=f \times_{M^{2}} \operatorname{id}_{M p^{c}(2 n, \mathbb{R})}: \tilde{P}_{1} \rightarrow \tilde{P}_{2}$ of principal $M p^{c}(2 n, \mathbb{R})$-bundles over $M$ as well as an automorphism $\bar{f}: P \rightarrow P$ of the principal $S p(2 n, \mathbb{R})$-bundle $P$ over $M$ taking $Q_{1} \subset P$ to $Q_{2} \subset P$, such that the diagram

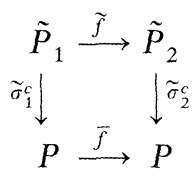

is commutative. Now it is known $[10,15]$ that this is not sufficient for the two $M p^{c}$-structures to coincide. However, any two $M p^{c}$-structures on $M$ differ by a uniquely determined element of the first sheaf cohomology group $H^{1}\left(M, C^{\infty}(M, U(1))\right)$ since this operates in a simply transitive manner on the set of $M p^{c}$-structures on $M$, and the previous argument shows that the two discussed above actually differ only by an element of $\operatorname{Im} d^{0}[10]$. Here, $d^{0}$ is the connecting homomorphism

$$
d^{0}: H^{0}\left(M, C^{\infty}(M, S p(2 n, \mathbb{R}))\right) \longrightarrow H^{1}\left(M, C^{\infty}(M, U(1))\right)
$$

in the long exact cohomology sequence induced from the short exact sequence

$$
1 \longrightarrow U(1) \longrightarrow M p^{c}(2 n, \mathbb{R}) \longrightarrow S p(2 n, \mathbb{R}) \longrightarrow 1
$$

of Lie groups.

Under the isomorphism $H^{1}\left(M, C^{\infty}(M, U(1))\right) \cong H^{2}(M, \mathbb{Z})$ given by the first Chern class, $\operatorname{Im} d^{0}$ is mapped to the 2-torsion subgroup of $H^{2}(M, \mathbb{Z})$. Thus if $H^{2}(M, \mathbb{Z})$ has no 2-torsion, the hermitian $M p^{c}$-structure on $M$ is independent of the 
compatible almost complex structure $J$ used in its definition and will therefore be called the universal one. Otherwise, we speak of the "universal" $M p^{c}$-structure on $M$ only if some choice of $J$ is understood. In any case, the hermitian connection depends on the choice of $J$.

\section{Applications to Geometric Quantization}

It is known that compatible almost complex structures on a symplectic manifold $(M, \omega)$ may be viewed as a particular case of Lagrangian vector subbundles of $T^{\mathbb{C}} M$ (also called polarizations if they satisfy additional requirements one of which is involutivity). More precisely, compatible almost complex structures $J$ bijectively correspond to those Lagrangian vector subbundles $F$ such that the restriction of the sesquilinear form $(X, Y) \mapsto-i \omega(\bar{X}, Y)$ to $F$ is positive definite. For short, such an $F$ is called positive definite, too. The correspondence assigns to $J$ its eigenbundle $F=T^{1,0} M$ for eigenvalue $i$; moreover, the latter is involutive if and only if the Nijenhuis torsion of $J$ vanishes. In addition, the eigenbundle $T^{0,1} M$ for eigenvalue $-i$ is $T^{0,1} M=\overline{T^{1,0} M}$ and satisfies $T^{1,0} M \oplus T^{0,1} M=T^{\mathbb{C}} M$.

Now the second author has proposed [9] a geometric quantization method (extending that of Kostant-Souriau) which consists of the following steps : First, a connection on the bundle of symplectic frames of $M$ is distinguished by a given pair of Lagrangian vector subbundles satisfying $F \oplus G=T^{\mathbb{C}} M$. This is done in a way very similar to that yielding the hermitian connection on an almost hermitian manifold. Then this connection is lifted to one on a suitable bundle of generalized metaplectic frames (of type $M p^{c}(2 n, \mathbb{R})$ ) such that the projection of its curvature to $u(1) \subset m p^{c}(2 n, \mathbb{R})$ is just $-2 \pi i \omega$. Next, using $F$ and $G$ once more, the structure group is reduced from $M p^{c}(2 n, \mathbb{R})$ to a suitable subgroup carrying a representation which allows to associate a complex line bundle $L$ with $2 c_{1}(L)=2[\omega]+c_{1}(M)$, where $[\omega] \in H^{2}(M, \mathbb{R})$ denotes the cohomology class of $\omega$. The sheaf of sections in $L$ which are covariant constant along $F$ serves to construct the quantizing Hilbert space in a manner entirely analogous to the Kostant-Souriau theory.

A complex line bundle $L$ with connection satisfying the above condition on its first Chern class will be called a quantum bundle - not to be confused with the prequantum bundle $L_{K S}$ introduced by Kostant [13] and Souriau [16] which has first Chern class $[\omega]$, if it exists.

Compared with the Kostant-Souriau theory, the underlying structure group $M p(2 n, \mathbb{R}) \times U(1)$ is replaced by its quotient $M p^{c}(2 n, \mathbb{R})$, allowing to abandon physically superfluous restrictions. Only the latter group has a direct physical interpretation, as it consists of all automorphisms of an irreducible Weyl system projecting down to the symplectic group (for this assertion, see [17], but note the different terminology used there).

In case the given Lagrangian vector subbundles satisfy $F \oplus G=T^{\mathbb{C}} M, G=\bar{F}$, and $F$ is positive definite, the above quantization method can be combined with the use of universal metaunitary and metaplectic structures. This choice of Lagrangian vector subbundles selects a reduction of the bundle of symplectic frames of $M$ from $\operatorname{Sp}(2 n, \mathbb{R})$ to $U(n)$, and under this reduction, the aforementioned connection distinguished by $F$ and $G$ is the hermitian connection with respect to the almost Kählerian structure on $M$ induced by $F$. 
Now consider diagram (2.13) and let $Q$ and $P$ be the unitary and symplectic frame bundles of $M$, respectively; further let $\tilde{Q}$ and $\tilde{P}$ be the universal metaunitary and metaplectic frame bundles of $M$, respectively. Since $Q$ and $P$ are equipped with the hermitian connection, we have to consider the hermitian connection on $\tilde{Q}$ and $\tilde{P}$ as well to obtain the following commutative diagram of principal bundles with connection

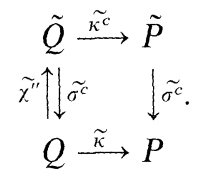

In the case of interest, $M U^{c}(n)$ is indeed a suitable subgroup of $M p^{c}(2 n, \mathbb{R})$. We have three characters of $M U^{c}(n)$ at our disposal, namely det ${ }^{\circ} \sigma^{c}, \eta$ and

$$
\begin{aligned}
\chi^{c}: M U^{c}(n) & \longrightarrow U(1) \\
{[A, \lambda] } & \longmapsto \chi(A) \cdot \lambda
\end{aligned}
$$

However, all three complex line bundles arising by associating $\mathbb{C}$ to $\tilde{Q}$ with one of these characters have first Chern class $c_{1}(M)$, and thus cannot be quantum bundles. But since $H^{2}(M, \mathbb{Z}) \cong H^{1}\left(M, C^{\infty}(M, U(1))\right)$ operates in a simply transitive manner on the equivalence classes of generalized metaunitary and metaplectic frame bundles (with structure groups $M U^{c}(n)$ and $M p^{c}(2 n, \mathbb{R})$, respectively) [10], this lack can be compensated for by twisting $\tilde{Q}$ and $\tilde{P}$ with the cohomology class $\frac{1}{2}\left(2[\omega]-c_{1}(M)\right)$ whenever the latter is integral. This will change the first Chern classes of the complex line bundles associated via det $\sigma^{c}, \eta$ and $\chi^{c}$ to $c_{1}(M), 2[\omega]$ and $\frac{1}{2}\left(2[\omega]+c_{1}(M)\right)$, respectively; thus the last one will be a candidate for a quantum bundle.

Let us make the twisting procedure more explicit, so that it also applies to the representing frame bundles and the connections thereon. The cohomology class $\frac{1}{2}\left(2[\omega]-c_{1}(M)\right)$ being represented by $\frac{1}{2}\left(2 \omega+\frac{1}{4 \pi} \varrho\right)$, choose a hermitian complex line bundle $K$ with connection such that its curvature is $\frac{1}{2}\left(-4 \pi i \omega-\frac{i}{2} \varrho\right)$, which is possible if and only if the above class is integral [13]. Then consider the homomorphisms

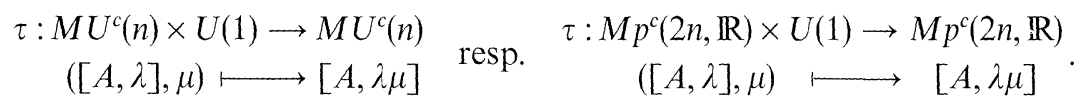

Applying these to (the transition functions of) the Whitney products $\tilde{Q} \times{ }_{M} \stackrel{\circ}{K}$ and $\tilde{P} \times{ }_{M} \stackrel{\circ}{K}$, where $\stackrel{\circ}{K}$ is the $U(1)$-frame bundle associated to $K$, we obtain new generalized metaunitary and metaplectic frame bundles $\tilde{Q}_{\omega}$ and $\tilde{P}_{\omega}$ (including lifting maps $\tilde{\sigma}_{\omega}^{c}: \tilde{Q}_{\omega} \rightarrow Q$ and $\tilde{\sigma}_{\omega}^{c}: \tilde{P}_{\omega} \rightarrow P$ ), respectively. Moreover, the hermitian connections on $\tilde{Q}$ resp. $\tilde{P}$ combine with that on $K_{K}^{\circ}$ to yield connections on $\tilde{Q}_{\omega}$ resp. $\tilde{P}_{\omega}$.

Finally, application of $\chi^{c}$ to (the transition functions of) the $M U^{c}(n)$-bundle $\tilde{Q}_{\omega}$ yields a principal $U(1)$-bundle $\stackrel{\circ}{L}$ with connection whose associated complex line bundle is the desired quantum bundle. In fact, it is just the quantum bundle considered in [6].

In contrast, application of $\eta$ to (the transition functions of) $\tilde{Q}_{\omega}$ or $\tilde{P}_{\omega}$ yields a principal $U(1)$-bundle $\stackrel{\circ}{L}_{K S}^{2}$ with connection whose associated complex line bundle 
is the square of the Kostant-Souriau prequantum bundle $L_{K S}$ whenever the latter exists.

The relations between the relevant principal bundles are summarized in the commutative diagram

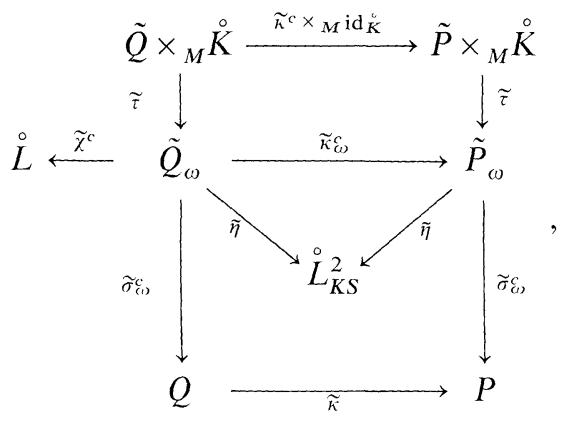

where all principal bundle morphisms are compatible with the respective connections.

Acknowledgements. The first author would like to acknowledge fruitful discussions with A. Back and P G O Fieund We would also like to thank the referee for clarifying suggestions

\section{References}

1. Atiyah, M.F.: Ann. Sci. Ec. Norm. Sup. 4, 47 (1971)

2. Atiyah, M.F., Bott, R., Patodi, V.K.: Inventiones Math. 19, 279 (1973); 28, 277 (1975)

3. Atiyah, M.F., Bott, R., Shapiro, A.: Topology 3, 3 (1964)

4. Back, A., Forger, M., Freund, P.G.O.: Phys. Lett. 77B, 181 (1978)

5. Borel, A.. Hirzebruch, F. Am J. Math 80. 458 (1958): 81. 315 (1959): 82. 491 (1960)

6. Czyz, J.: On geometric quantization and its connections with the Maslov theory. Preprint, University of Warsaw (1977)

7. Geroch, R. : J. Math. Phys. 9, 1739 (1968); 11, 343 (1970)

8. Hawking, S., Pope, C. N. : Phys. Let1. 73 B, 42 (1978)

9. Hess, H.: Forthcoming thesis

10. Hess, H., Krausser, D.: Lifting classes of principal bundles. Rep. Math. Phys. (to appear)

11. Hitchin, N.J.: Adv. Math. 14, 1 (1974)

12. Kobayashi, S., Nomizu, K.: Foundations of differential geometry, Vol. 1 and 2. New York: Interscience 1963/1969

13. Kostant, B.: Quantization and unitary representations. In: Lectures in modern analysis and applications III; Lecture notes in mathematics, Vol. 170. Berlin, Heidelberg, New York: Springer 1970

14. Kostant, B.: Symplectic spinors. In: Convegno di geometria simplettica e fisica matematica, INDAM, Rome (1973); Symp. Math. 14. New York: Academic Press 1974

15. Milnor, J.: L'Ens. Math. 9, 198 (1963)

16. Souriau, J.M.: Commun. math. Phys. 1, 374 (1966); see also: Structure des systèmes dynamiques. Paris: Dunod 1969

17. Weil, A.: Acta Math. 111, 143 (1964)

18. Whiston, G.S. : Gen. Relativ. Grav. 6, 463 (1975)

Communicated by R. Geroch 